\title{
Duck! Scaling the height of a horizontal barrier to body height
}

\author{
Jeanine K. Stefanucci and Michael N. Geuss \\ University of Utah, Salt Lake City, Utah
}

\begin{abstract}
Recent research shows that the body is used to scale environmental extents. We question whether the body is used to scale heights as measured by real actions (Experiments 1 and 2) or by judgments about action and extent made from a single viewpoint (Experiments 3 and 4). First, participants walked under barriers naturally, when wearing shoes, or when wearing a helmet. Participants required a larger margin of safety (they ducked at shorter heights) when they were made taller. In follow-up experiments, participants visually matched barrier heights and judged whether they could walk under them when wearing shoes or a helmet. Only the helmet decreased visually matched estimates; action judgments were no different when participants' eye height increased. The final experiment suggested that the change in matched estimates may have been due to lack of experience wearing the helmet. Overall, the results suggest that perceived height is scaled to the body and that when body height is altered, experience may moderate the rescaling of height.
\end{abstract}

Gibson (1979) hypothesized that perception was directly related to action. Specifically, actors can directly perceive what the environment affords them (affordances). What an environment affords an individual is determined by the characteristics of the environment (height, width, etc.) and by the capability of the actor. For example, a doorway affords walking under upright if the height of the doorway is sufficiently higher than the upright height of the actor.

Some research has demonstrated that people can perceive whether the width of an aperture affords passage. Warren and Whang (1987) found that participants scaled their decision of whether an aperture afforded passage to the width of their shoulders. Specifically, when the ratio of aperture-to-shoulder width was 1.16 or larger, participants indicated that they could pass through an aperture. Wagman and Taylor (2005) found that participants who were asked to hold or view objects of different sizes when anticipating walking through an aperture adjusted their judgments of passability to take into account the size of the object. Ishak, Adolph, and Lin (2008) also investigated participants' ability to judge whether they could reach into an aperture to retrieve candy. Their findings showed that participants scaled their decisions to their hand size. Ishak et al. also varied the size of the hand by placing a prosthesis on participants. The participants, in turn, adjusted their reachability judgments accordingly, showing that they were sensitive to the new size of their hand.

Although much has been done to assess how people perceive whether they can fit through an aperture, fewer studies have determined how people perceive whether they can walk under a barrier. Perceiving whether an aperture affords passing under is reliant on perceiving the height of the aperture or barrier in relation to the height of the body. We will first review the literature that describes how people normatively estimate heights, and then we will discuss the literature that suggests that a certain aspect of the body, eye height, seems to be the most likely reference for deciding whether one can pass under a barrier or not.

\section{Normative Estimation of Heights}

Sinai, Ooi, and He (1998) found that participants who stood at the top of heights ( $\sim 6 \mathrm{ft}$ or $2 \mathrm{~m}$ in size) overestimated the vertical distance to the ground by about 50\% (see also Jackson \& Cormack, 2007; Stefanucci \& Proffitt, 2009, for more overestimation of taller heights). They concluded that this overestimation was likely due to a misperception of eye height, but they did not include a situation in which participants viewed the height from the ground. Yang, Dixon, and Proffitt (1999) also tested perception of the height of short objects (such as a light pole or a door) and found approximately $6 \%$ overestimation. Their results suggested that taller objects (buildings) were overestimated more than were shorter objects (doors), even when the visual angle to all of the objects from the viewing position of the observer was held constant. Overall, many studies have demonstrated that heights are overestimated and that larger heights are overestimated more than shorter heights; however, no one has looked at the normative estimation of heights that can be acted on (e.g., walking underneath).

\section{Scaling Perceived Height}

It is possible that for shorter heights (or those in the range of standing height of the participants), observers

J. K. Stefanucci, jeanine.stefanucci@psych.utah.edu 
will reference their body size to scale the extent of the height more accurately. Previous research has shown that people scale visually matched estimates of spatial layout to their bodies. For example, Witt, Proffitt, and Epstein (2005) showed that the perception of distance in near or peripersonal space (the area defined as reachable and just beyond; see Cutting \& Vishton, 1995) was scaled to the length of the participants' arms. When participants held a baton, which extended the space within which they could reach targets, targets that were formerly out of reach appeared closer. Linkenauger, Witt, Stefanucci, Bakdash, and Proffitt (2009) have also shown that the perceived distance to an object and the perceived size of an object is related to the arm length and the hand size of the participant. Participants who perceived their arms as longer also perceived targets as closer and the space within which they could reach objects as larger. Likewise, participants who estimated their hand to be larger also reported that objects appeared smaller. Similarly, Stefanucci and Geuss (2009) have shown that perceived aperture width, outside near space, is related to the perceived size of the body. They found that wider participants estimated aperture widths to be smaller than did narrower participants. In addition, when participants held a large object or held out their arms, they judged apertures to be smaller than did participants who did not hold an object or whose arms were by their sides.

More important for this article, van der Meer (1997) showed that both tall $(>190 \mathrm{~cm})$ and short $(<175 \mathrm{~cm})$ adults who walked under barriers of varying heights ducked at a height that was 1.04 times their standing height, whereas children ducked at a height that was 1.11 times their standing height. Recent work has shown that people who viewed a horizontal barrier from a static viewpoint also scaled their action judgments (estimates of whether they could successfully walk under the height without ducking) to their own height (Wagman \& Malek, 2008). In more recent work, Wagman and Malek (2009) measured participants' willingness to pass under barriers of different heights that they anticipated walking or running under (although they never truly performed these actions). Not surprisingly, they found that participants were more willing to walk than to run under such barriers, especially those that were tall. Willingness to pass under a barrier was thus influenced by the anticipated speed of passage and the height of the barrier relative to participant height. Further research has shown that participants' judgments about whether they could pass under a lintel when performing a novel action suggests that practice with the novel action (e.g., wheeling a wheelchair) can increase the accuracy of the judgments (Stoffregen, Yang, Giveans, Flanagan, \& Bardy, 2009). Wagman and Malek (2008) also found that participants' action judgments were altered slightly by the location of their eye height (in contrast to anticipated speed, as manipulated in Wagman \& Malek, 2009) when viewing a horizontal barrier from a nonmoving viewpoint. For example, when participants were sitting on the ground or standing on a stool, their judgments of whether they could walk under a barrier were less cautious than when they were standing on the ground. Although they argued that the change in action judgments was due to participants' needing to complete a series of nested goals before estimating whether they could pass under, we believe that the change in action judgments could have been due to changes in eye height.

Many researchers have found that observers can scale the sizes or heights of targets to their eye height (Bertamini, Yang, \& Proffitt, 1998; Dixon, Wraga, Proffitt, \& Williams, 2000; Sedgwick, 1973; Wraga, 1999). Sedgwick was the first to propose this idea, and he outlined the mechanism by which participants could use eye height information when standing on the ground plane to scale the sizes of objects that were also on the ground plane, regardless of their distance to the object. Testing this notion further, Bertamini et al. found that observers were better at judging the heights of objects when the sizes of the objects were in the range of the eye height of the participant, whether seated or standing. For example, the height of an object was more accurately perceived to be its height when the observer was standing and the object's size was approximately the standing eye height of the participant. Wraga also showed that manipulating the height at which participants viewed an object could influence their estimates of the size of the object. Specifically, she used a false floor to present objects to participants either at the normal floor level or at $17 \mathrm{~cm}$ above the normal level (effectively reducing eye height information for participants). When the floor was raised, participants overestimated the height of the objects, as predicted, by approximately $14 \%$. Dixon et al. extended these findings by showing that manipulations of the location of an observer's eye height using virtual reality resulted in a change in their estimates of the height of objects in the virtual world. Participants estimated the objects to be larger when the center of projection of the virtual scene was placed at 0.7 times actual eye height (unbeknownst to participants) than when the center of projection was placed at actual eye height.

Eye height is also used to make action judgments in the environment, such as whether a surface affords sitting or climbing (Mark, 1987) and whether a doorway affords passage (Warren \& Whang, 1987). For example, Warren and Whang employed a raised floor and found that participants in that condition judged narrower apertures as passable compared with participants who viewed the apertures on a normal floor. Mark strapped 10-cm blocks onto the feet of participants in order to raise their eye height. He found that participants' judgment of the maximum seat height that they perceived as "sittable" was greater when wearing the blocks and that the maximum step height that they perceived as "climbable" was shorter.

\section{The Present Study}

Overall, previous findings suggest that participants scale the heights of objects to their eye height. Few studies have tested the perception of the height of objects for which observers can walk under, however. Furthermore, many of the affordance judgments obtained in the previous work did not involve real interaction with the heightrather, a judgment of "passability" or "sittability" from a static viewpoint only. We added to this literature by first 
asking participants to walk under a horizontal barrier to find the ratio of participant height to the height of a barrier under which participants would walk without ducking. To our knowledge, this action boundary has been documented in only one previous study (van der Meer, 1997). We documented this boundary in order to compare it with manipulations of physical height and eye height, as well as to gauge when people decide to duck in order to walk under an object.

After identifying this action boundary for a horizontal barrier, we then changed the dimensions of the body to discern whether these bodily changes influenced participants' ducking behaviors (Experiment 2). We manipulated the height of participants in two ways to test whether eye height scaling was present. One manipulation included changing eye height by having participants stand on blocks, a manipulation similar to that of Mark (1987). The other manipulation involved participants donning a helmet, thereby changing the height of their body but not their eye height. In this manner, we were able to test for differences in action boundaries when a change to the eye height of the observer was present versus not present. We expected participants to be more cautious walking under (ducking at lower heights) when they are made taller but that the caution might be greater when there is no change to eye height because they may be less sure of their physical height.

In Experiments 3 and 4, we obtained both action judgments and visual estimates of the height of the barrier from participants who viewed the barrier from a static position. In this manner, we expanded on previous work by relating visual estimates of the vertical extent to affordance judgments of passability. We believe that visually matched estimates of the height that are taken from a static viewing position may inform later interactions with the height, as well as being influenced by changes to the body; however, manipulations of eye height may affect decisions about actions in a different manner than they affect matched estimates of the height. For example, in order to decide whether a barrier affords walking under, one might simulate walking under the barrier in order to make the decision. This simulation may not be necessary to match a different extent to the height of the barrier, however. Again, we assessed the influence of a change to the body on the matched estimates of height and the static affordance judgments to discern whether manipulations of the body similarly or differentially affect these perceptual estimates. Finally, we suspected that participants would be cautious both in their judgments of action from a single viewpoint (obtained in Experiments 3 and 4A) and in their actual actions observed in Experiments 1 and 2. Very few studies have directly compared action judgments with real actions (see, e.g., Richardson, Marsh, \& Baron, 2007), so we hope to be able to compare these measures for perceiving heights as well.

\section{EXPERIMENT 1}

The purpose of this experiment was to determine the point at which upright walking transitions into bent walk- ing or crouching when participants have to walk under a barrier. Participants were videotaped walking underneath a horizontal barrier (a PVC pipe) positioned at different heights. The tallest barrier height for which participants bent to walk under was recorded. This height and the participant's height determined the action boundary for each participant. We determined this boundary by scaling the highest passable height to the participant's height, which created a ducking point relative to physical height. This allowed for a comparison of boundaries across participants of different physical heights (short and tall). We predicted that there would be no difference between tall and short individuals' judgments if their actions were scaled to their own heights (as observed by van der Meer, 1997).

\section{Method}

Participants. Ten (4 female, 6 male) volunteers participated in the study. Volunteers were recruited via e-mails and flyers during a summer session. All participants were naive to the purposes of the experiment and gave written informed consent to participate. All participants came to the experiment wearing comfortable shoes for walking.

Apparatus. All judgments were made in a $304.8 \times 304.8 \mathrm{~cm}$ room with a solid colored wall. A horizontal barrier was constructed by suspending a pipe ( $152.4 \mathrm{~cm}$ long, $2.54 \mathrm{~cm}$ in diameter) from the ceiling. The height of the barrier was adjustable through a series of pulleys. The barrier was placed $152.4 \mathrm{~cm}$ in front of the starting position, which was $114.3 \mathrm{~cm}$ from a wall opposite the barrier. The barrier was raised and lowered to 13 different test heights (147.3$208.3 \mathrm{~cm}$, in $5.08-\mathrm{cm}$ intervals).

Participants walking underneath the horizontal barrier were recorded using a JVC Everio GZ-MG330 video camera in the back corner of the room that was mounted on a tripod $182.88 \mathrm{~cm}$ from the barrier (see Figure 1). The participants' standing height and eye height were recorded with a standard $12-\mathrm{ft}(3.63-\mathrm{m})$ tape measure.

Procedure. Participants were instructed to walk naturally underneath the barrier and to bend as needed to avoid collision. Once they passed under the barrier, they were instructed to stop, make a $180^{\circ}$ turn, and return back under. Each participant completed a trial at

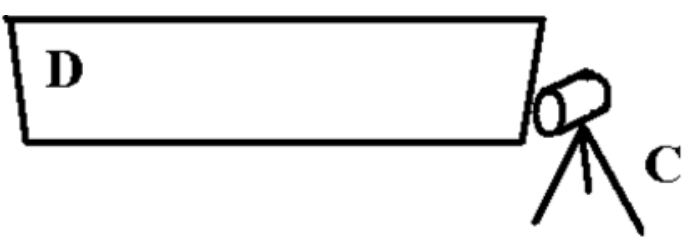

$\mathbf{A}$

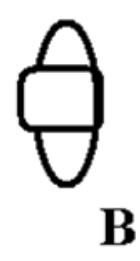

Figure 1. Overhead view of the experimental setup for all experiments. (A) The horizontal barrier, which was raised and lowered to different heights. (B) The location of the participant during static judgments and the starting position during action trials. (C) The approximate position of the camera used in Experiments 1 and 2. (D) The location of the projection screen in Experiments 3 and $4 \mathrm{~A}$. 
each test height 3 times, resulting in 78 passages in total. Test heights were presented in random order.

After the participants returned to the wall opposite the barrier, they remained facing away from the barrier. The next aperture height was set while the participants faced the wall. The experimenter moved out of view before the participants turned to face the barrier again. This process was repeated for each test height. When all 78 passages had been completed, the participant's height was recorded.

\section{Results}

Videos were analyzed by two coders who were blind to the pole's height on each trial. The coders recorded any ducking or bending behavior for each trial. Bending behavior was defined a priori as any motion by any part of the body (head, neck, torso, or knees) that resulted in a lowered height of the participant. Interrater reliability was $97.69 \%$.

Average height of participants. A median split on the standing height of participants was conducted to form the tall and short groups. The average height of the short group (3 female, 2 male) was $165.86 \mathrm{~cm}(S D=4.4)$. The average height of the tall group (1 female, 4 male) was $183.26 \mathrm{~cm}(S D=7.1)$. Females had an average height of $168.51 \mathrm{~cm}$, and males had an average height of $178.60 \mathrm{~cm} .{ }^{1}$ The average height of all participants was $174.56 \mathrm{~cm}(S D=10.8)$. An independent-samples $t$ test confirmed that the tall group was significantly taller than the short group $[t(8)=-4.58, p=.002]$.

Action boundaries. To create an action boundary, we divided the barrier height at which participants ducked $50 \%$ of the time by the participant's height. This scaled the required environmental extent (the barrier height) to each actor's height. The mean ratio of barrier height to participant height was 1.03 , with a standard error of 0.01 . Action boundaries ranged from 0.99 to 1.09. In other words, participants required, on average, a barrier height that was 1.03 times their own height to walk under without bending.

Independent-samples $t$ tests were run to compare action boundaries between height groups. The analysis revealed no significant differences between height groups $[t(8)=$ $0.29, p=.68]$. This tentatively suggests that tall and short individuals require relatively the same amount of space above their heads to walk under a horizontal barrier without ducking.

\section{Discussion}

In this experiment, participants were asked to walk under different barrier heights and to duck as needed to avoid collision. As expected, we found that taller individuals ducked at taller barrier heights. When barrier heights were scaled to participant height, however, we found no differences in action boundaries across the tall and short groups. These results suggest that participants scaled barrier heights to their own height when walking underneath the barrier. The barrier must have been at least 1.03 times their height, on average, for them to walk under while standing upright (or, on average, $5.24 \mathrm{~cm}$ taller than their height). This ratio matched almost exactly that obtained by van der Meer (1997). A ratio that is greater than 1 suggests that participants allowed for a margin of safety above their head when passing under an object, as they did in Warren and Whang (1987) when passing through an aperture.

\section{EXPERIMENT 2}

In this experiment, we sought to replicate the results of Experiment 1, but we also wanted to discern whether participants would scale the height of a barrier to their body when their body height had been altered. We therefore added to participants' height by either placing a helmet on their head or asking them to stand on blocks of wood and attaching them to their feet. As in Experiment 1, participants walked under horizontal barriers of different heights. We hypothesized that when wearing an object, the observer would require a larger margin of safety when passing under the barrier.

\section{Method}

Participants. Ten (3 female, 7 male) undergraduates from the College of William \& Mary participated in the study for course credit (mean age $=21.5$ years). All participants were naive to the purpose of the experiment and gave written informed consent to participate.

Apparatus. All aspects of the barrier and the video recording were the same as those in Experiment 1. The barrier was raised and lowered to 10 different test heights $(152.4-198.12 \mathrm{~cm}$, in $5.08-\mathrm{cm}$ intervals). For one height manipulation, participants were asked to don an ROTC helmet that increased their height by approximately $2.54 \mathrm{~cm}$. A chin strap secured the helmet on their head for the duration of the experiment, and a net inside suspended it at a consistent height above the head. It is important to note that the helmet did not obscure or block the field of view of participants in any way (as a baseball cap might). For the other height manipulation, participants were asked to strap wooden blocks that were $3.18 \mathrm{~cm}$ tall onto the bottom of their shoes with Velcro. All participants were told to wear tennis shoes when signing up for the experiment to ensure some consistency across this manipulation and to reduce the chances that participants would show up in high heels.

Procedure. Participants were instructed to walk naturally underneath the barrier and to bend as needed to avoid collision. Once they passed under the barrier, they were instructed to stop, make a $180^{\circ}$ turn, and return back under. Participants walked under each of the 10 heights twice when wearing the helmet, twice when wearing the blocks, and twice not wearing either. The order of the object conditions was such that the no-object condition was always second. The helmet and blocks conditions alternated between being either first or third for participants. After all blocks of trials were completed, the participant's true height was recorded, as well as their height when wearing the helmet and the blocks.

\section{Results}

Videos were analyzed by two coders who were blind to the pole's height on each trial. The coders recorded any ducking or bending behavior for each trial across blocks (helmet, no object, or blocks). Bending behavior was defined a priori as any motion by any part of the body (head, neck, torso, or knees) that resulted in a lowered height of the participant. Interrater reliability was $94 \%$.

Average height of participants in each condition. The average height of the group when wearing the helmet was $174.85 \mathrm{~cm}(S D=2.4)$. The average height of the group when wearing the blocks was $176.53 \mathrm{~cm}(S D=$ 
2.6). The average height of all participants when not wearing any object was $172.22 \mathrm{~cm}(S D=2.6)$.

Action boundaries. Because we had homogeneous groups (participants were in all conditions and height distribution therefore did not change among the conditions), we did not analyze ducking behavior in addition to determining action boundaries, as was done in Experiment 1; analyzing the action boundary would yield the same results as would comparing ducking behavior across the conditions. To create action boundaries for each height condition, we divided the previously used barrier heights by the participant's height for the noobject condition, by the participant's height when wearing the helmet for the helmet condition, and by the participant's height when wearing the blocks for the blocks condition. This scaled the required environmental extent (the barrier height) to the actor's current height when walking underneath the barrier. The mean ratio of barrier height to participant height in the no-object condition was 1.002 , with a standard error of 0.006 . Action boundaries ranged from 0.99 to 1.02 . In other words, participants required, on average, a barrier height that was 1.002 times their own height to walk under without bending. Their actions were quite accurate. The mean ratio of barrier height to participant height when wearing the helmet in the helmet condition was 1.03 , with a standard error of 0.011 . Action boundaries ranged from 1.006 to 1.06 . The mean ratio of barrier height to participant's height when wearing the blocks in the block condition was 1.02 , with a standard error of 0.009 . Action boundaries ranged from 0.99 to 1.04 .

A 2 (order) $\times 3$ (object: helmet, blocks, none) $\times 2$ (direction: forward, return) repeated measures ANOVA was run to compare action boundaries across participant height manipulations. The analysis revealed a significant effect of object $\left[F(2,9)=9.54, M S_{\mathrm{e}}=.001, p=.002, \eta_{\mathrm{p}}^{2}=.54\right]$. There was no effect of direction $[F(1,8)=1.18, p=.31]$ or order $[F(1,8)=1.12, p=.32]$.
Post hoc analyses using Fisher's LSD revealed that participants were more cautious when wearing a helmet than when wearing no object. Participants were also more cautious when wearing blocks than when wearing no object. Action boundaries were no different for wearing the helmet than for wearing the blocks ( $p=.19$; see Figure 2 ).

\section{Discussion}

In this experiment, participants were asked to walk under different barrier heights and to duck as needed to avoid collision. They walked under the heights when wearing a helmet, when wearing blocks on their feet, and when not wearing any object. As expected, when participants were wearing an object that made them taller, they required taller barrier heights to pass under without ducking. Analyses revealed that when this barrier height was scaled to the participants' increased heights, participants required a larger margin of safety when wearing an object (helmet or blocks) than when not wearing an object. In addition, participants were less conservative in their ducking behavior when not wearing an object. Although comparisons across experiments were not made, participants in this experiment seemed less conservative than those in the previous experiment (action boundary of 1.002 vs. 1.03). We believe that participants in this experiment may have been less conservative because they always performed the no-object condition after an object condition; when participants were not wearing an object, they might have been relieved from being overly cautious (which they were when wearing an object) and so acted less cautious than participants in Experiment 1, who had not just removed an object. Overall, the results suggest that when a change was made to the height of participants, they allowed for a larger margin of safety when walking under the barrier. In Experiments 3 and 4A, we investigated whether participants needed a larger margin of error because they estimated the barrier height as shorter when changes were made to their height.

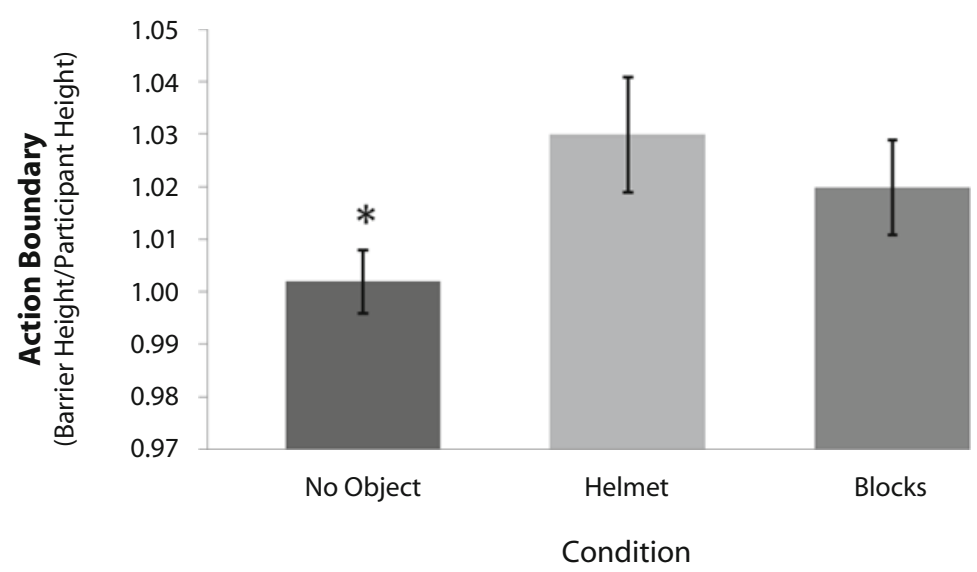

Figure 2. Action boundaries are represented as the barrier height at which participants transitioned from upright walking to "ducking" divided by participant height. The no-object condition required a significantly smaller margin of safety than did the helmet and blocks conditions. Error bars represent one standard error. 


\section{EXPERIMENT 3}

Previous work has shown that altering the body (by placing weights on the ankles) can result in changes to participants' judgments of their jumping ability and the jumping ability of others (Ramenzoni, Riley, Shockley, \& Davis, 2008). In this experiment, we investigated whether manipulating participants' heights would influence their judgment of the height of a horizontal barrier as well as their judgment about whether they could pass under it upright. Specifically, we added objects (the wooden blocks used in Experiment 2) to participants' feet. In contrast to Experiments 1 and 2, participants did not walk under the barrier. Rather, participants in this experiment completed two perceptual judgments from a static viewing point (although participants were allowed to sway, and they probably gained some information from that; see Mark, Balliett, Craver, Douglas, \& Fox, 1990; Stoffregen, Yang, \& Bardy, 2005). We questioned whether participants who wore the blocks would (1) scale action decisions made from one viewpoint to their new height and (2) estimate the barrier to be shorter with a visual matching task when wearing the blocks. We predicted that participants would indicate that they required a larger margin of safety when wearing the blocks on their feet. Additionally, we expected that when participants wore the blocks they would visually estimate the height of the barrier to be shorter than would those who did not wear the blocks.

\section{Method}

Participants. Thirty-eight (17 female, 21 male) College of William \& Mary students participated in the experiment for credit in an introductory psychology course. All participants were naive to the purpose of the experiment and gave written informed consent to participate.

Apparatus. Visually matched estimates were obtained by having participants replicate the extent of the height (from the floor to the barrier) in a horizontal dimension. To do this, participants were asked to adjust two lines that were projected onto a large projection screen $(177.8 \mathrm{~cm}$ high $\times 210.82 \mathrm{~cm}$ wide) which stood $76.2 \mathrm{~cm}$ from the floor in the experiment room. An NEC NP60 projector was connected to a Dell Inspiron 1521 laptop running MATLAB, which was used to display the adjustable lines to participants. Participants made half of their height judgments while wearing wooden blocks attached to their feet (the same as those described in Experiment 2). The blocks added $3.18 \mathrm{~cm}$ to the height of the participants. All other apparati were the same as those in the previous experiments.

Design. A mixed design was used. All participants were in both conditions (blocks and no blocks) when making the affordance judgments, so the manipulation of height was within subjects for the affordance judgments. Order of condition was randomized and counterbalanced among participants. Participants saw each barrier height twice when wearing the blocks and twice when not wearing them. Nine heights were presented, ranging from 157.48 to $198.12 \mathrm{~cm}$, in increments of $5.08 \mathrm{~cm}$ (or $2 \mathrm{in}$.). The nine barrier heights were blocked and randomized within each block (so that two blocks of nine heights were presented for each condition: blocks and no blocks). The result was 18 affordance judgments per condition per participant.

A between-subjects design was used to collect the visually matched estimates of the heights, however. After making all affordance judgments, participants visually estimated each of the nine heights. For the block of visually matched estimates, participants viewed the heights in the same condition as that used in the previous block of affordance trials (either with blocks or without blocks). For example, if participants made affordance judgments wearing the blocks first and then made judgments not wearing the blocks, then they visually matched the heights when not wearing the blocks as well.

Procedure. Participants made two perceptual judgments about the height of the horizontal barrier. Participants stood at the home position $139.7 \mathrm{~cm}$ from the horizontal barrier. They judged passage under the barrier and estimated its height from this point. They were instructed to imagine walking underneath the horizontal barrier without bending or ducking in any way to make their affordance judgments.

Participants gave four judgments of passage for each of the nine heights. Two judgments were made in each condition (blocks, no blocks). Participants were instructed to respond (yes or no) whether they thought they could walk under the barrier without ducking. In the blocks condition, participants were asked to strap the blocks to their shoes with Velcro. In this condition, participants were instructed to make all judgments assuming that they would walk under the barrier with the blocks attached to their feet.

Visually matched estimates of height were always made at the end of the last block of affordance judgments. Again, the condition for the last block of affordance judgments (either blocks or no blocks) was the condition that was used for the matched estimates. To complete the visual matching task, participants matched the height of the barrier to the distance between two vertical bars projected onto a solid projector screen. At the start of each trial, the vertical bars were projected at a random distance from each other. The participant then (via instructions to the experimenter, who hit the up or down arrow on the laptop keyboard) moved the bars closer together or farther apart until the horizontal distance between the bars was the best representation of the vertical height of the barrier. The experimenter sat out of participants' view and adjusted the distance between the bars per the participant's instructions. Participants were encouraged to look back and forth between the barrier height and the horizontal distance between the bars as many times as needed until they were satisfied that it best represented the vertical extent between the floor and the barrier. Visually matched estimates of height were made for all nine heights in random order. The participant was instructed to turn around between each presentation of height and thus never saw the experimenter near the projection screen or the barrier.

\section{Results}

Affordance judgments. Transition points were computed for both blocks of trials for both conditions (blocks and no blocks) for each participant. In both cases, the transition barrier height was computed by averaging the lowest height the participant indicated he or she could pass under without ducking and the highest height that the participant indicated he or she could not pass under. The transition point for the head condition was computed by dividing the transition barrier height by the participant's height (top of the head to the floor). The transition point for the blocks condition was computed by dividing the transition barrier height by the participant's height when wearing the blocks (top of the head to the bottom of the blocks). The result was four transition points per participant, two for each block within each condition.

A 2 (order: blocks first, head first) $\times 2$ (condition: blocks, no blocks) $\times 2$ (blocks of trials) repeated measures ANOVA comparing transition points, with order as the only between-subjects factor, revealed a significant main effect of condition $\left[F(1,41)=4.82, M S_{\mathrm{e}}=.001\right.$, $\left.p=.03, \eta_{\mathrm{p}}^{2}=.11\right]$. Participants needed a larger margin of safety to pass under when they wore the blocks $(M=$ 


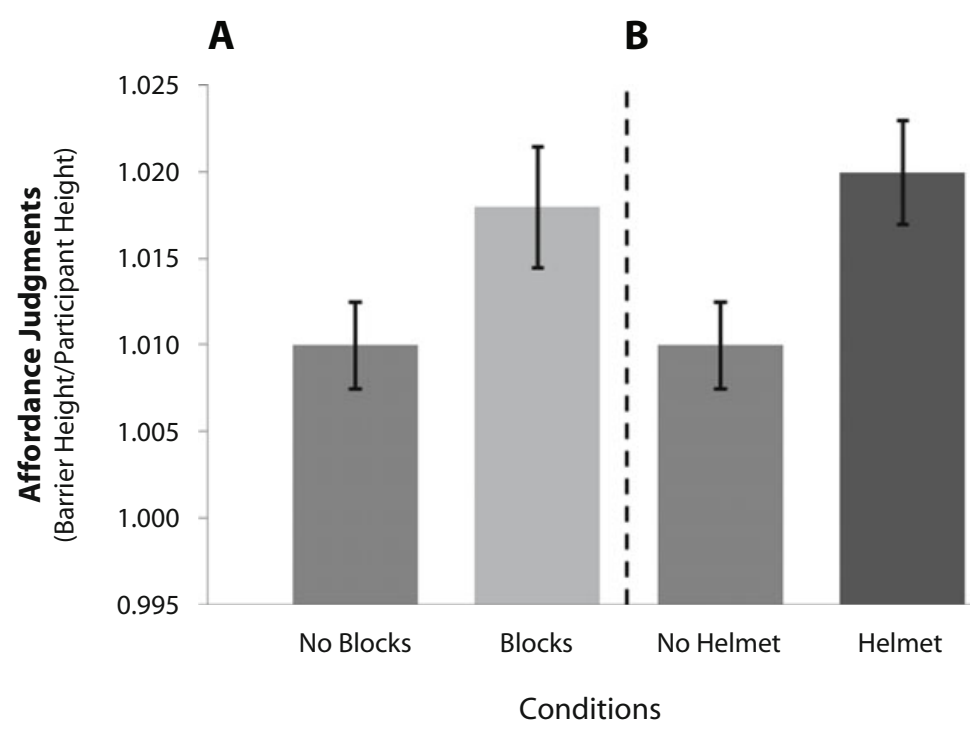

Figure 3. Affordance judgments displayed as the average barrier height that marked the transition from indicating passability to not divided by participant height. (A) Affordance data shown for the no-blocks and blocks conditions from Experiment 3. (B) Affordance data shown for the no-helmet and helmet conditions from Experiment 4A. Error bars represent one standard error.

$1.018, S E=0.007)$ than they needed when they wore no object $(M=1.01, S E=0.005$ (see Figure $3 \mathrm{~A})$. There was no significant effect of trial block $[F(1,27)=0.01, p=$ $.92]$ or order $[F(1,41)=1.03, p=.32]$.

Height estimates. A 2 (condition: blocks, no blocks) $\times$ 9 (barrier height) repeated measures ANOVA comparing estimates of height, with condition as a between-subjects factor and barrier height as a within-subjects factor, did not reveal a significant main effect of condition $[F(1,36)=$ $0.18, p=.68]$. Estimations of the height of the barrier were no different among participants wearing the blocks
$(M=214.61, S E=4.62)$ than among the participants who were not wearing the blocks $(M=217.37, S E=4.62)$ (see Figure 4). As expected, there was a significant main effect of barrier height $\left[F(8,288)=68.76, M S_{\mathrm{e}}=228.98, p<\right.$ $\left..0001, \eta_{\mathrm{p}}^{2}=.73\right]$.

\section{Discussion}

The results indicated that changing the height of participants by adding blocks under their feet influenced participants' judgments about their action capabilities. Participants' affordance judgments indicated that they would

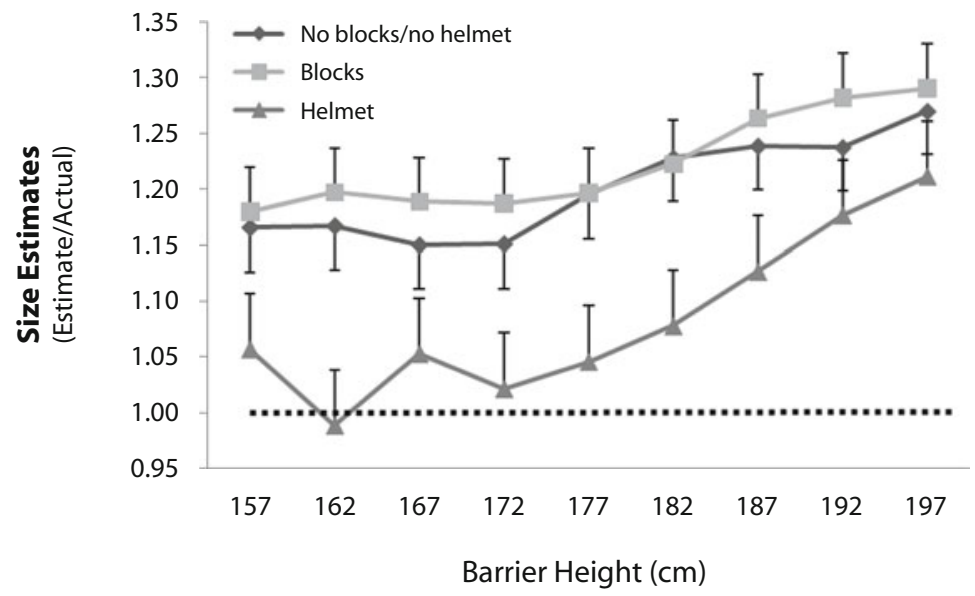

Figure 4. Ratios of estimate of height to actual barrier height were computed for each barrier height. In Experiment 4A, participants' estimates of the barrier height were shorter than their estimates when wearing no object. Wearing blocks under one's feet did not significantly influence estimations of barrier height. In all conditions, participants overestimated the height of the barrier. Estimations of height during the no-blocks and no-helmet conditions in Experiments 3 and $4 \mathrm{~A}$, respectively, were averaged for display purposes only. 
be more cautious walking under the barrier when wearing the blocks than when wearing no object. These results are consistent with the findings of Experiment 2, in which participants were more cautious walking under the barrier when wearing the same blocks. We did not see any differences in size estimates between participants who wore the blocks and those who did not wear the blocks, however. This finding could be the result of a definite change in the eye height of participants, which they used to rescale perceived height. Also, given that the height of the blocks was smaller than the increments that differentiated the barrier heights, the change may have been too small to alter size estimates. Our finding may also be due to participants' experience with changes to their height under their feet. At this point, however, we cannot make any definitive conclusions about the impact of experience on size judgments. Overall, participants did overestimate the height with the visually matched estimates, as we would have expected from previous research (Stefanucci \& Proffitt, 2009).

\section{EXPERIMENT 4A}

In this experiment, we investigated whether manipulating participants' heights without altering eye height would also influence their judgments of the height of horizontal barriers. Specifically, we added an object (the army helmet used in Experiment 2) to participants' heads instead of adding an object to their feet as we had done in Experiment 3. We wondered whether participants would adequately scale decisions about actions to their own height when they wore an object that changed their physical height, but not their eye height. Participants, especially females, likely have more experience with changes to height that occur when wearing shoes of different heights. Manipulations of height that occur above the eyes may be harder to take into account, given that participants have less practice with these manipulations. In this experiment, participants made two perceptual judgments about the barrier's height, the same judgments that participants in Experiment 3 made: a judgment about passage, and then a visually matched estimate of the barrier's height. We predicted that participants would report feeling less able to pass under the barriers when wearing a helmet than when not wearing a helmet. Additionally, we expected that when participants wore the helmet, they would visually estimate the height of the barrier to be shorter than they would when not wearing the helmet.

\section{Method}

Participants. Twenty-nine (18 female, 11 male) College of William \& Mary students participated in the experiment for credit in an introductory psychology course. All participants were naive to the purpose of the experiment and gave written informed consent to participate.

Apparatus. Visually matched estimates were obtained in the same manner as that used in Experiment 3: by having participants replicate the extent of the height (from the floor to the barrier) in a horizontal dimension on a projection screen. Participants made half of their height judgments while wearing an army helmet. The helmet was borrowed from the local ROTC chapter, and it added
$2.54 \mathrm{~cm}$ to the height of the participants (the same helmet as that used in Experiment 2). All other apparati were the same as those in previous experiments.

Design. The design was the same as that of Experiment 3. A mixed design was used; all participants were in both conditions (helmet, no helmet) when making affordance judgments. After participants completed two blocks of trials of affordance judgments (one for each condition), they were asked to complete the visually matched estimates of height for whichever condition they had performed second. The visually matched estimates were thus obtained between subjects.

Procedure. The procedure was exactly the same as the procedure in Experiment 3. Participants gave two perceptual judgments about the height of a horizontal barrier: affordance judgments and visually matched estimates of height. Participants stood at the home position $139.7 \mathrm{~cm}$ from the horizontal barrier. They judged passage under the barrier and estimated the barrier's height from this point. They were instructed to imagine walking underneath the horizontal barrier without bending or ducking in any way to make their affordance judgments.

In the helmet condition, participants were asked to don the army helmet. The straps of the helmet were pulled snugly underneath the chin to keep the helmet stable. In the helmet condition, participants were instructed to make all judgments while keeping in mind the height of the helmet in addition to their own height.

\section{Results}

Affordance judgments. Transition points were computed for both blocks of trials and for both conditions (helmet and no helmet) for each participant. In both conditions, the barrier height was computed by averaging the lowest height the participant indicated he or she could pass under without ducking and the highest height that the participant indicated he or she could not pass under. The transition point for the head condition was computed by dividing the barrier height by the participant's height (top of the head to the floor). The transition point for the helmet condition was computed by dividing the barrier's height by the participant's height when wearing the helmet (top of the hat to the floor). The result was four transition points per participant, two for each block within each condition.

A 2 (order: helmet first, head first) $\times 2$ (condition: helmet, no helmet) $\times 2$ (blocks of trials) repeated measures ANOVA comparing transition points, with all factors within subjects, revealed a significant main effect of condition $\left[F(1,28)=7.63, M S_{\mathrm{e}}<.000, p=.01, \eta_{\mathrm{p}}^{2}=\right.$ .21]. Participants needed a larger margin of safety to pass under when they wore the helmet $(M=1.02, S E=0.006)$ than they needed when they did not wear a helmet $(M=$ $1.01, S E=0.005$ ) (see Figure 3B). There was no significant effect of trial block $[F(1,28)=0.55, p=.46]$ or order $[F(1,28)=1.20, p=.28]$.

Height estimates. A 2 (condition: helmet, no helmet) $\times$ 9 (barrier height) repeated measures ANOVA comparing estimates of height, with condition as a between-subjects factor and barrier height as a within-subjects factor, revealed a significant main effect of condition $[F(1,27)=$ $\left.4.83, M S_{\mathrm{e}}=6,262.05, p=.037, \eta_{\mathrm{p}}^{2}=.15\right]$. Participants wearing the helmet estimated the height of the barrier to be shorter $(M=192.71, S E=7.05)$ than did participants who wore no object $(M=214.25, S E=6.81)$ (see Fig- 
ure 4). There was also a significant main effect of barrier height $\left[F(8,216)=60.78, M S_{\mathrm{e}}=303.8, p<.0001\right.$, $\left.\eta_{\mathrm{p}}^{2}=.69\right]$.

\section{Discussion}

Participants responded that they required a larger amount of space above the helmet to pass under the barrier than they required above their head to pass under the barrier. The results showed that altering participants' height by adding an object to the top of the head influenced how participants scaled their action judgments. These results are consistent with how participants acted in Experiment 2 when wearing the same helmet.

In addition, participants who wore the helmet visually estimated the barrier to be shorter than did participants who did not wear the helmet. This result suggests that when body height is altered while not manipulating eye height, estimates of the extent may change in addition to a change in action judgments. We suspect that the effect on perceptually matched estimates is due, in part, to participants' lack of experience with altering their height by adding objects to their head. Specifically, seeing the height as smaller when wearing the helmet may have served as an initial warning mechanism to participants whose height is not normally altered in this manner. To test this claim, we examined the influence of altering one's height on the perception of barrier heights with a population of "experienced" hat wearers - specifically, members of the local ROTC chapter.

\section{EXPERIMENT 4B}

Many studies have found that experience performing an action, such as passing through an aperture, may not be necessary in order for judgments of action capability to be rescaled when dimensions of the body have been altered (Higuchi, Takada, Matsuura, \& Imanaka, 2004; Savelsbergh, Douwes Dekker, Vermeer, \& Hopkins, 1998). Judgments of whether or not one can pass under a lintel when in a wheelchair, however, become more accurate with only 2 min of practice wheeling the chair (Stoffregen et al., 2009). The results of Experiment 3 suggest that changes to eye height - specifically, raising eye height with an addition to the shoes - may not rescale visually matched judgments of height. This null result could have been due to participants' knowledge about the change to their eye height or to their experience with wearing shoes of different heights. The purpose of this experiment was to determine whether experience with changes to body height that occur on the head could alter visually matched estimates of height and judgments of passage. Specifically, do the effects of seeing the barrier as shorter and acting as if it is shorter when wearing the helmet dissipate with experience? To test the effect of experience, participants were recruited from the local ROTC chapter-individuals who wear the same army helmet as that used in Experiments 2 and $4 \mathrm{~A}$ an average of $7 \mathrm{~h}$ per week. We thought that people who were enrolled in ROTC might show differences in their judgments of passage and in their perceptual estimates given their experience wearing the helmet.

\section{Method}

Participants. Eleven (4 female, 7 male) College of William \& Mary ROTC students were paid $\$ 5$ for their participation in the experiment. All participants were naive to the purpose of the experiment and gave written informed consent to participate.

Procedure. All other apparati, design, and procedures were identical to those used in Experiment 4A.

\section{Results}

We were unable to collect 1 participant's estimates of height because of a technical error with the laptop. Unfortunately, no more than 11 participants were willing to participate from the ROTC chapter; therefore, we consider these results to be preliminary. We also conducted more qualitative analyses in addition to the quantitative analyses, given the limited number of participants and likely lower power for detecting effects.

Affordance judgments. Transition points were computed for both helmet and head conditions using the same method as that in Experiment 4A. Once again, the result was four transition points per participant, two for each block within each condition.

A 2 (order: helmet first, head first) $\times 2$ (condition: helmet, no helmet) $\times 2$ (blocks of trials) repeated measures ANOVA comparing transition points, with order as the only between-subjects factor, revealed a significant main effect of condition $\left[F(1,9)=6.44, M S_{\mathrm{e}}<.000, p=.03\right.$, $\left.\eta_{\mathrm{p}}^{2}=.42\right]$. Participants needed a larger margin of safety to pass under when they wore the helmet $(M=1.02, S E=$ $0.01)$ than they needed when they did not wear a helmet $(M=1.00, S E=0.007)$. There was no significant effect of trial block $[F(1,9)=0.20, p=.67]$ or order $[F(1,9)=$ $0.06, p=.82]$. Qualitatively, closer inspection of the data showed that 9 of the 11 participants were more cautious when wearing the helmet than when not wearing the helmet, which obviously drove the significance that we obtained in the analysis.

Height estimates. A 2 (condition: helmet, no helmet) $\times$ 9 (barrier height) repeated measures ANOVA comparing estimates of height, with condition as a between-subjects factor and barrier height as a within-subjects factor, revealed no main effect of condition $[F(1,8)=0.651, p=$ $\left..44, \eta_{\mathrm{p}}^{2}=.07\right]$. Estimates of barrier height when participants wore the helmet $(M=216.51, S E=10.14)$ were not different from estimates made when they were not wearing the helmet $(M=228.08, S E=10.14)$. As expected, there was a significant main effect of barrier height $[F(8,64)=$ $\left.24.76, M S_{\mathrm{e}}=262.25, p<.0001, \eta_{\mathrm{p}}^{2}=.76\right]$. Qualitatively, closer inspection of these data showed that estimates for 3 of the 5 participants in the helmet condition trended in the opposite-than-predicted direction, so that they judged the barrier to be taller when wearing the helmet. Similarly, 2 of the 5 participants in the head condition had estimates trending in the opposite-than-predicted effect; specifically, all of their estimates were shorter than average.

\section{Discussion}

People with experience wearing the ROTC helmet anticipated needing a larger margin of safety for passage under a barrier when wearing the helmet than when not wearing the helmet. This result is consistent with the af- 
fordance judgments of Experiment 4A among participants who, we assume, had limited experience wearing helmets, and it is also consistent with the real actions performed by participants in Experiment 2. It seems that even with experience, participants allow for a larger margin of safety in making action judgments from a nonmoving viewpoint when wearing a helmet than when not.

The limited data in this experiment also suggest, however, that the experienced ROTC participants may not have estimated the height to be shorter, as the inexperienced helmet participants did in Experiment 4A. These findings suggest that the perception of barrier heights as shorter when wearing the helmet may dissipate with practice, although more research is needed to verify this with a larger sample. We do believe that the small effect size obtained for the visually matched estimates (7\%) suggests that the effect in experienced helmet wearers, if it does exist, may be smaller than that observed in inexperienced participants. Future studies could train "novice" helmet wearers on scaling heights while wearing the helmet to determine whether there is a true pretest-posttest difference when training is more controlled.

\section{GENERAL DISCUSSION}

Two interesting and novel findings come out of this work. First, we identified the height at which participants will begin to duck when walking under a horizontal barrier (an action boundary for ducking) and verified that this boundary is extremely similar to that observed by van der Meer (1997). Moreover, this action boundary can be altered if participants' eye height or physical height is manipulated. In addition, not only are real actions affected by these changes to eye height and physical height, but action judgments from a single viewpoint (here termed affordance judgments) are rescaled with changes to the height of the body. The second, potentially more interesting finding of these experiments is that they show that visually matched estimates of the height of the barrier, unlike affordance judgments, may not always be rescaled with changes to height. Specifically, the matched estimates were rescaled when physical height was changed without altering eye height (helmet) but not when eye height was also changed (blocks). These experiments do support previous findings that heights are overestimated when measured with visually matched estimates of height (Stefanucci \& Proffitt, 2009); however, these experiments show that this overestimation can occur even with extents that are in the range of the standing height of the participant. We will discuss these findings and their implications more below. Overall, this series of experiments showed that people scale the height of a horizontal barrier to their own height, but that the rescaling may differ on the basis of whether real actions, action judgments, or visually matched judgments are used to detect changes in perception.

The results from Experiment 1 demonstrate that when participants walked underneath a barrier, they required the height of the barrier to be 1.03 times their own height in order to walk under without ducking. This result adds to the findings of Warren and Whang (1987), who docu- mented that when participants walked through a doorway, they required the width of the doorway to be 1.3 times the width of their shoulders in order to walk through without rotating their shoulders. Warren and Whang claimed that 1.3 times the shoulder width of the observer allowed for a margin of safety that would be needed in order to avoid collision. The findings of Experiment 1 in the present article suggest that participants may need a smaller margin of safety when walking under a barrier (1.03) than when walking through an aperture (1.3; as also observed by van der Meer, 1997). Although reasons for this disparity were not tested, we believe that this difference in margin of safety could be due to greater amounts of "sway" (side-toside) that occur when walking as compared with "bounce" (vertical sway) produced when walking. If bounce is not as great as sway, then the margin of safety needed to walk under an object without ducking could be reduced in comparison with walking through an aperture. Another possibility is that rotating the shoulders to avoid collision can occur in only one dimension - rotating the shoulders around the vertical axis - whereas ducking can occur by moving the head or bending the knees. A larger margin of safety may therefore be needed when only one dimension can be rotated or altered to avoid collision.

The other important set of findings, which come mainly out of Experiments 3, 4A, and 4B, suggests that when viewed from a nonmoving viewpoint, perceived barrier height may be rescaled as a result of manipulations of the height of the body; however, the measure of perceived height that was used seemed to contribute to whether an effect was observed. This work employed two perceptual measures of height: affordance judgments and visually matched estimates of height. In all cases, a change in height resulted in a change in action judgments, which we expected given Wagman and Malek's (2008) findings that eye height could influence affordance judgments and Ramenzoni et al.'s (2008) findings that wearing ankle weights can rescale participants' judgments of jumping ability. The affordance judgments also mirrored changes in real actions that were observed in Experiment 2, so that when participants were taller they reported being less likely to pass under from a nonmoving viewpoint and they were more likely to duck when actually passing under. This supports other findings in which real action-scaled ratios have been similar to action-judgment ratios (see Richardson et al., 2007). When participants' heights were changed by having them wear blocks under their shoes, however, their visually matched estimates of the height were not different from those who did not wear blocks. This finding is interesting in that it represents a situation in which visually matched estimates were not rescaled because of changes in eye height, as one would expect on the basis of the previous research on eye height scaling of size (Bertamini et al., 1998; Dixon et al., 2000; Sedgwick, 1973; Wraga, 1999). Some important differences in our experimental design could explain this discrepancy. First, in the previous research on eye height scaling of size, changes were often made to the environment, usually outside of the awareness of the participants, in order to manipulate perceived eye height (e.g., with virtual reality or with a false 
floor). Our participants were clearly aware of a change in height, because it occurred on the person. Given that they could also see the extent of the change (the blocks) in some conditions, this could have informed their estimates. Further work is needed to determine whether awareness of the change could influence visually matched estimates. Also, in the previous work on eye height scaling of size, vision was often restricted, and participants were able to use only visual cues, such as the level of the horizon, to estimate the height or size of objects. Our experiments were conducted in a full-cue environment; thus, in the case of the blocks, other information could have supplemented the obvious change in eye height to result in similar scaling of heights across conditions (wearing the blocks or not). When eye height was not changed but physical height was (as in the case of the helmet), however, participants not only adjusted their action decisions to be more cautious but also reported seeing the barrier as shorter.

There are myriad reasons why there could be a discrepancy in visually matched estimates across experiments other than the issue of awareness. An obvious limitation is that changes to the body using the blocks and the helmet were not conducted within subjects; therefore, differences across the participants could have led to our null result. There is no reason to believe that the populations of participants were drastically different, however, given that the affordance judgments were similar across experiments. Also, it is possible that changes to eye height are more quickly integrated into the visual system, which results in an immediate change to perceptual judgments. Participants wearing the hat may have needed more time to adjust to their new physical height and to rescale visually matched estimates. In other words, it is possible that the change in visually matched estimates is due to a lack of experience with changes to physical height that are not accompanied by a change in eye height. This explanation is partially supported by Stoffregen et al.'s (2009) finding that experience wheeling a wheelchair made later judgments about passage under a lintel more accurate, even when the practice did not involve wheeling under barriers.

The results of Experiments 4A and 4B suggest that experience may influence the rescaling of perceived height, although these conclusions are tentative given the small sample obtained for Experiment 4B. Participants in Experiment $4 \mathrm{~A}$, presumably with less experience wearing helmets, reported seeing the aperture as shorter when wearing the helmet than did experienced participants in Experiment 4B. Furthermore, this finding may reflect a warning system that exaggerates to the inexperienced participant that he or she is taller and needs to be more cautious, especially when he or she has not acted in the environment. Although the number of ROTC members who were able to participate was small, the findings, along with those of Experiment 3, provide tentative support for the notion that experience with changes to the body may influence visually matched estimates of height. This tentative moderating effect of experience may be especially important when the changes that are made to the body do not influence eye height or are not easily incorporated through some other mechanism.
Future research could investigate the possible moderating influence of experience on size estimates. We believe that fewer people experience changes to their height that involve adding objects to the head than those experiencing changes that involve adding objects to the feet. Obviously, both occur in daily life (wearing high heels or wearing a tall hat), but the occurrence of changes to the feet may be more common. To further test the notion that experience with changes to bodily height moderates height perception, it would be interesting and useful to test other populations of people who are more experienced with changes to their height in future studies. For example, people who wear stilts would likely estimate a height differently than would someone who has never worn stilts. Pilots may be more adept at estimating height given their level of experience with judging heights. Also, participants could be trained with changes to height, and their visually matched estimates could be observed over the course of the training to reveal the point at which experience alters matching estimates. As shown by Stoffregen et al. (2009), the training may not need to be extensive. Other lines of research have also shown that practice learning a skill can alter actions and perception (Bruggeman, Pick, \& Rieser, 2005; Repp $\&$ Knoblich, 2007). Bruggeman et al. showed that participants recalibrated their throwing behavior over time when asked to throw to a target while on a rotating carousel. Repp and Knoblich found that trained pianists were more likely to hear a tone pair as rising when their actions (playing the piano keys) were consistent with that perception.

Another important question to address would be whether the level of change in bodily height contributed directly to the extent of rescaling observed in these experiments. We made only small changes to the body and observed fairly small but significant changes in action judgments and sometimes in visually matched estimates of height. This rescaling of height perception was not surprising, given that we have shown that changes in the body's width can rescale the perception of the width of apertures (Stefanucci \& Geuss, 2009). It would be useful to identify how large the change in bodily height needs to be to result in a rescaling of perceived height, however. Furthermore, it would be interesting to assess whether changes to bodily height influence perception in a linear fashion, or whether there is a general threshold at which a change to the body initiates a warning mechanism and alters perceived barrier height. In our previous work on changes to body width resulting in a rescaling of perceived aperture size, we found that once participants' bodies were made wider than their shoulders, participants perceived the size of the aperture to be smaller (Stefanucci \& Geuss, 2009).

Similarly, it is unclear whether small changes in the nature of these experiments would affect the scaling of large heights, like a building, which have been the focus of most of our previous work (Stefanucci \& Proffitt, 2009; Stefanucci \& Storbeck, 2009; Teachman, Stefanucci, Clerkin, Cody, \& Proffitt, 2008). We would not predict a rescaling of tall heights when changes to the body are small, but if someone were standing on stilts or holding a long pole upright, then rescaling might arise. Further work is needed to explore the conditions under which rescaling 
may occur. The findings of Ramenzoni et al. (2008) suggest that simply adding ankle weights to participants was sufficient to change participants' judgments about jumping ability for heights that were larger than those used in this experiment.

Overall, our work suggests that the body, particularly eye height, is used to scale the extent of heights that one anticipates walking under. The findings add to a growing body of literature that has found that the body affects perceptual estimates of passage through an aperture. Here, we find that the body may also influence some (but not all) perceptual estimates of passage under an aperture and estimates of the height of the aperture. Furthermore, these estimates may be influenced by the level of experience that observers have with changes to their eye height.

\section{AUTHOR NOTE}

This research was supported in part by NSF Grant IIS-0914488, for which the first author is a co-PI, and NIH Grant RO1MH075781-01A2, for which the first author serves as a consultant. The authors thank Andrea Bidanset and Laura Shepherd for their help in collecting the data. Correspondence concerning this article should be addressed to J. K. Stefanucci, Department of Psychology, University of Utah, 380 South 1530 East, Room 502, Salt Lake City, UT 84112-0251 (e-mail: jeanine .stefanucci@psych.utah.edu).

\section{REFERENCES}

Bertamini, M., Yang, T. L., \& Proffitt, D. R. (1998). Relative size perception at a distance is best at eye level. Perception \& Psychophysics, 60, 673-682.

Bruggeman, H., Pick, H. L., JR., \& Rieser, J. J. (2005). Learning to throw on a rotating carousel: Recalibration based on limb dynamics and projectile kinematics. Experimental Brain Research, 163, 188197.

Cutting, J. E., \& Vishton, P. M. (1995). Perceiving layout and knowing distances: The integration, relative potency, and contextual use of different information about depth. In W. Epstein \& S. J. Rogers (Eds.), Handbook of perception and cognition: Vol. 5. Perception of space and motion (pp. 69-117). San Diego: Academic Press.

Dixon, M. W., Wraga, M., Proffitt, D. R., \& Williams, G. C. (2000). Eye height scaling of absolute size in immersive and nonimmersive displays. Journal of Experimental Psychology: Human Perception \& Performance, 26, 582-593.

Gibson, J. J. (1979). The ecological approach to visual perception. Boston: Houghton Mifflin.

Higuchi, T., Takada, H., Matsuura, Y., \& ImanaKa, K. (2004). Visual estimation of spatial requirements for locomotion in novice wheelchair users. Journal of Experimental Psychology: Applied, 10, 55-66.

IshaK, S., AdolPh, K. E., \& LiN, G. C. (2008). Perceiving affordances for fitting through apertures. Journal of Experimental Psychology: Human Perception \& Performance, 34, 1501-1514.

JACKSON, R. E., \& CORMACK, L. K. (2007). Evolved navigation theory and the descent illusion. Perception \& Psychophysics, 69, 353-362.

Linkenauger, S. A., Witt, J. K., StefanUcCi, J. K., BaKdash, J. Z., \& Proffitt, D. R. (2009). Effects of handedness and reachability on perceived distance. Journal of Experimental Psychology: Human Perception \& Performance, 35, 1649-1660.

MARK, L. S. (1987). Eyeheight-scaled information about affordances: A study of sitting and stair climbing. Journal of Experimental Psychology: Human Perception \& Performance, 13, 361-370.

Mark, L. S., Balliett, J. A., Craver, K. D., Douglas, S. D., \& Fox, T. (1990). What an actor must do in order to perceive the affordance for sitting. Ecological Psychology, 2, 325-366.

Ramenzoni, V. C., Riley, M. A., Shockley, K., \& Davis, T. (2008).
Carrying the height of the world on your ankles: Encumbering observers reduces estimates of how high an actor can jump. Quarterly Journal of Experimental Psychology, 61, 1487-1495.

RePP, B. H., \& KNOBLICH, G. (2007). Action can affect auditory perception. Psychological Science, 18, 6-7.

Richardson, M. J., Marsh, K. L., \& Baron, R. M. (2007). Judging and actualizing intrapersonal and interpersonal affordances. Journal of Experimental Psychology: Human Perception \& Performance, 33, 845-859.

Savelsbergh, G. J. P., Douwes Dekker, L., Vermeer, A., \& HopKINS, B. (1998). Locomoting through apertures of different width: A study of children with cerebral palsy. Pediatric Rehabilitation, 2, $5-13$.

SEDGwICK, H. A. (1973). The visible horizon: A potential source of visual information for the perception of size and distance. Dissertation Abstracts International, 34, 1301-1302B. (UMI No. 73-22, 530)

SinAI, M. J., OoI, T. L., \& He, Z. J. (1998). Terrain influences the accurate judgment of distance. Nature, 395, 497-500.

StefanUCCI, J. K., \& Geuss, M. N. (2009). Big people, little world: The body influences size perception. Perception, 38, 1782-1795.

Stefanucci, J. K., \& Proffitt, D. R. (2009). The roles of altitude and fear in the perception of height. Journal of Experimental Psychology: Human Perception \& Performance, 35, 424-438.

Stefanucci, J. K., \& Storbeck, J. (2009). Don't look down: Emotional arousal elevates height perception. Journal of Experimental Psychology: General, 138, 131-145.

Stoffregen, T. A., YAng, C.-M., \& Bardy, B. G. (2005). Affordance judgments and nonlocomotor body movement. Ecological Psychology, 17, 75-104.

Stoffregen, T. A., Yang, C.-M., Giveans, M. R., Flanagan, M., \& BARDY, B. G. (2009). Movement in the perception of an affordance for wheelchair locomotion. Ecological Psychology, 21, 1-36.

Teachman, B. A., Stefanucci, J. K., Clerkin, E. M., Cody, M. W., \& ProffitT, D. R. (2008). A new mode of fear expression: Perceptual bias in height fear. Emotion, 8, 296-301.

VAN DER MEER, A. L. H. (1997). Visual guidance of passing under a barrier. Early Development \& Parenting, 6, 149-157.

Wagman, J. B., \& Malek, E. A. (2008). Perception of affordances for walking under a barrier from proximal and distal points of observation. Ecological Psychology, 20, 65-83.

Wagman, J. B., \& MALEK, E. A. (2009). Geometric, kinetic-kinematic, and intentional constraints influence willingness to pass under a barrier. Experimental Psychology, 56, 409-417.

Wagman, J. B., \& TAYLOR, K. R. (2005). Perceiving affordances for aperture crossing for the person-plus-object system. Ecological Psychology, 17, 105-130.

WARREN, W. H., \& Whang, S. (1987). Visual guidance of walking through apertures: Body-scaled information for affordances. Journal of Experimental Psychology: Human Perception \& Performance, 13, 371-383.

Witt, J. K., Proffitt, D. R., \& Epstein, W. (2005). Tool use affects perceived distance, but only when you intend to use it. Journal of Experimental Psychology: Human Perception \& Performance, 31, 880-888.

Wraga, M. (1999). Using eye height in different postures to scale the heights of objects. Journal of Experimental Psychology: Human Perception \& Performance, 25, 518-530.

Yang, T. L., Dixon, M. W., \& Proffitt, D. R. (1999). Seeing big things: Overestimation of heights is greater for real objects than for objects in pictures. Perception, 28, 445-467.

\section{NOTE}

1. We decided not to perform analyses of gender, because gender analyses would have resulted in similar findings, given that membership in a height group covaried with gender.

(Manuscript received December 3, 2009; revision accepted for publication March 1, 2010.) 